PROCEEDINGS OF THE

AMERICAN MATHEMATICAL SOCIETY

Volume 138, Number 2, February 2010, Pages 557-565

S 0002-9939(09)10155-7

Article electronically published on September 30, 2009

\title{
USING ALEKSANDROV REFLECTION TO ESTIMATE THE LOCATION OF THE CENTER OF EXPANSION
}

\author{
YU-CHU LIN AND DONG-HO TSAI \\ (Communicated by Chuu-Lian Terng)
}

\begin{abstract}
We use the Aleksandrov reflection result of Chow and Gulliver to show that the center of expansion in expanding a given convex embedded closed curve $\gamma_{0} \subset \mathbb{R}^{2}$ lies on a certain convex plane region interior to $\gamma_{0}$.
\end{abstract}

\section{INTRODUCTION}

The purpose of this paper is to first see that, under a mild assumption on the speed function (the function $G$ in (1) below), there exists a center of expansion when we expand a convex embedded closed curve $\gamma_{0} \subset \mathbb{R}^{2}$ to infinity. In [T2] we first discussed its definition and established its existence under a stronger assumption of the speed function. However, if the center exists, we do not know in general whether it is enclosed by the initial curve $\gamma_{0}$ or lies outside $\gamma_{0}$.

In this paper, we shall apply the Aleksandrov reflection result of Chow-Gulliver CG to show that this center of expansion actually lies on a certain convex plane region interior to $\gamma_{0}$.

Let $\gamma_{0}$ be a smooth convex closed curve in $\mathbb{R}^{2}$ parametrized by a smooth embed$\operatorname{ding} X_{0}(\alpha): S^{1} \rightarrow \mathbb{R}^{2}$. Consider the expanding flow

$$
\left\{\begin{array}{l}
\frac{\partial \gamma}{\partial t}(\alpha, t)=G\left(\frac{1}{k(\alpha, t)}\right) \cdot \mathbf{N}(\alpha, t), \quad \alpha \in S^{1}, \quad t>0, \\
\gamma(\alpha, 0)=X_{0}(\alpha), \quad \alpha \in S^{1},
\end{array}\right.
$$

where $\mathbf{N}(\alpha, t)$ is the unit outward normal to $\gamma(\alpha, t), k(\alpha, t)$ is the curvature of $\gamma(\alpha, t)$, and $G:(0, \infty) \rightarrow(0, \infty)$ is a given smooth function with $G^{\prime}>0$ everywhere (parabolicity condition). The function $G$ determines the speed of the flow.

By [CT], it is known that the flow (11) has a convex smooth solution $\gamma(\alpha, t), \alpha \in$ $S^{1}$, defined on some maximal time interval $\left[0, T_{\max }\right), T_{\max } \leq \infty$, where the maximum curvature $k_{\max }(t)$ is decreasing on $\left[0, T_{\max }\right)$ and $k(\cdot, t)$ tends to zero uniformly as $t \rightarrow T_{\max }$. The curve $\gamma(\cdot, t)$ then expands to infinity in a uniform way as $t \rightarrow T_{\max }$.

It is also known (see Urbas [U]) that, in terms of the support function $u(\theta, t)$ of $\gamma(\cdot, t)$, where the parameter $\theta \in S^{1}$ represents the outward normal angle, the flow

Received by the editors August 4, 2008.

2000 Mathematics Subject Classification. Primary 35K15, 35K55.

The research of the second author was supported by NSC (grant number 95-2115-M-007-009) and the research center NCTS of Taiwan.

(C)2009 American Mathematical Society Reverts to public domain 28 years from publication 
(11) is equivalent to the following scalar equation:

$$
\left\{\begin{array}{l}
\frac{\partial u}{\partial t}=G\left(u_{\theta \theta}+u\right), \quad \theta \in S^{1}, \quad t>0, \\
u(\theta, 0)=u_{0}(\theta)>0, \quad \theta \in S^{1} .
\end{array}\right.
$$

Here we have assumed, without loss of generality, that $\gamma_{0}$ encloses the origin of $\mathbb{R}^{2}$ so that the initial support function $u_{0}(\theta)$ is positive everywhere. Moreover, the curvature $k_{0}(\theta)$ of $\gamma_{0}$ can be expressed as

$$
\frac{1}{k_{0}(\theta)}=\left(u_{0}\right)_{\theta \theta}(\theta)+u_{0}(\theta)>0 \text { for all } \theta \in S^{1} .
$$

Since $S^{1}$ is compact, there exists a positive constant $\delta>0$ such that $\left(u_{0}\right)_{\theta \theta}(\theta)+$ $u_{0}(\theta) \geq \delta>0$ for all $\theta \in S^{1}$. By the maximum principle, we also have

$$
u_{\theta \theta}(\theta, t)+u(\theta, t) \geq \delta>0 \quad \text { for all } \quad(\theta, t) \in S^{1} \times\left[0, T_{\max }\right) .
$$

We can then use the support function $u(\theta, t)$ to construct a family of convex (due to (4) ) closed plane curves satisfying the expanding flow (11).

For the solution $u(\theta, t)$ to (2), it is also proved in [CT that $u_{\theta}(\theta, t)$ and $u_{\theta \theta}(\theta, t)$ are both uniformly bounded (independent of time) on $S^{1} \times\left[0, T_{\max }\right.$ ), with bounds depending only on the initial data $u_{0}(\theta)$.

We refer the reader to the paper by Andrews $\mathrm{A}$ and the references cited therein for contemporary research results on various kinds of curvature flow of plane curves. In particular, the paper by Gage-Hamilton [GH] is a classic.

From now on, in addition to the basic assumption that $G:(0, \infty) \rightarrow(0, \infty)$ is smooth with $G^{\prime}>0$ everywhere, we further assume the following:

Main assumptions on $G$ : We assume $(* 1): \lim _{z \rightarrow \infty} G(z)=\infty$, and $(* 2)$ : for any constant $C>0$, there exists a constant $\lambda>0$ such that

$$
0<\frac{1}{\lambda} \leq \frac{G^{\prime}(\xi)}{G^{\prime}(z)} \leq \lambda \quad \text { for all } \xi \in[z-C, z+C]
$$

as long as $z$ is large enough.

Remark 1. For convenience, we shall use $(* 1)$ and $(* 2)$ to denote each assumption respectively. Examples for $G(z)$ satisfying $(* 1)$ and $(* 2)$ include $G(z)=$ $z^{\alpha}, G(z)=[\log (z+1)]^{\alpha}, G(z)=e^{\alpha z}$, where $\alpha>0$ is any constant, and many more.

Remark 2 (observed by the referee). One can check that if there exists a number $z_{0}>0$ so that $\log G^{\prime}(z)$ is uniformly continuous on $\left[z_{0}, \infty\right)$, then condition $(* 2)$ is satisfied. The above three examples of $G(z)$ all satisfy it.

As pointed out in [T2], there exists a solution $R(t)$ to the ODE $d R / d t=G(R(t))$ on $\left[0, T_{\max }\right)$ which satisfies

$$
0<u_{\min }(t) \leq R(t) \leq u_{\max }(t) \quad \text { for all } t \in\left[0, T_{\max }\right) .
$$

Moreover, the condition $\lim _{z \rightarrow \infty} G(z)=\infty$ will imply that such an $R(t)$ is unique. The three functions $u_{\min }(t), R(t)$, and $u_{\max }(t)$ all tend to infinity as $t \rightarrow T_{\max }$, with $0 \leq u_{\max }(t)-u_{\min }(t) \leq c$ for some constant $c$ independent of time (since $u_{\theta}(\theta, t)$ is uniformly bounded on $\left.S^{1} \times\left[0, T_{\max }\right)\right)$. 


\section{AleKSANDROV REFLECTION AND CENTER OF EXPANSION}

Under assumptions $(* 1)$ and $(* 2)$, we can guarantee the existence of the center of expansion.

Theorem 3. Assume $(* 1),(* 2)$ and that the convex closed initial smooth curve $\gamma_{0}$ encloses the origin. Then under the flow (1),$\gamma(\cdot, t)$ will remain smooth and convex and will expand to infinity as $t \rightarrow T_{\max } \leq \infty$. Moreover, the support function $u(\theta, t) \in C^{\infty}\left(S^{1} \times\left[0, T_{\max }\right)\right)$ of $\gamma(\cdot, t)$ satisfies the estimate

$$
\lim _{t \rightarrow T_{\max }}\|u(\theta, t)-(R(t)+a \cos \theta+b \sin \theta)\|_{C^{1}\left(S^{1}\right)}=0
$$

for some constant $(a, b) \in \mathbb{R}^{2}$.

Remark 4. As defined in [T2], we call $(a, b) \in \mathbb{R}^{2}$ the center of expansion of $\gamma(\cdot, t)$. (77) says that asymptotically $u(\theta, t)$ has no higher mode of Fourier series coefficients.

Remark 5. The geometric meaning of (17) is that as $\gamma(\cdot, t)$ expands to infinity, its support function is close to that of the expanding circle $C(\cdot, t)$, where $C(\cdot, t)$ is centered at $(a, b) \in \mathbb{R}^{2}$ with radius $R(t), t \in\left[0, T_{\max }\right)$. By (13) below, $\gamma(\theta, t)$ and $C(\theta, t)$ have position vectors close to each other at every $\theta \in S^{1}$. In particular the Hausdorff distance (see Lemma 15 below) between $\gamma(\cdot, t)$ and $C(\cdot, t)$ tends to zero as $t \rightarrow T_{\max }$. This justifies the terminology of the center of expansion.

Remark 6. It is known from [T2 that, under the flow (1), the isoperimetric difference $L^{2}(t)-4 \pi A(t)$ of the evolving curves is always decreasing on $t \in\left[0, T_{\max }\right)$. Moreover if the flow (11) has a center of expansion, then the isoperimetric difference decreases to zero. Here $L(t)$ is the length of $\gamma(\cdot, t)$ and $A(t)$ is its enclosed area. Hence if $L^{2}(t)-4 \pi A(t)$ does not decrease to zero, the flow will not have a center of expansion. We will need this observation later on.

Proof. One can prove Theorem 3 by following arguments similar to those in T2. We will be rather sketchy here. Set $w(\theta, t)=u(\theta, t)-R(t),(\theta, t) \in S^{1} \times\left[0, T_{\max }\right)$, where $R(t)$ is from (6). Its evolution can be written as

$$
\begin{aligned}
\frac{\partial w}{\partial t}(\theta, t) & =a(\theta, t)\left[w_{\theta \theta}(\theta, t)+w(\theta, t)\right], \\
a(\theta, t) & =\int_{0}^{1} G^{\prime}\left(s\left(w_{\theta \theta}+w\right)(\theta, t)+R(t)\right) d s>0 .
\end{aligned}
$$

Since $G^{\prime}(z)$ may tend to 0 or $+\infty$ as $z \rightarrow \infty, a(\theta, t)$ may approach 0 or $+\infty$ as $t \rightarrow T_{\max }$, which prevents equation (8) from being uniformly parabolic. To make it uniformly parabolic, we rescale time by setting $\tau(t)=\log [G(R(t)) / G(R(0))] \in$ $[0, \infty), t \in\left[0, T_{\max }\right)$. We have $\tau\left(T_{\max }\right)=\infty$ (since $G$ satisfies $\left.(* 1)\right)$, which is crucial. In terms of $\tau, w(\theta, \tau)$ satisfies

$$
\left\{\begin{array}{l}
\frac{\partial w}{\partial \tau}(\theta, \tau)=A(\theta, \tau)\left[w_{\theta \theta}(\theta, \tau)+w(\theta, \tau)\right], \quad(\theta, \tau) \in S^{1} \times[0, \infty) \\
A(\theta, \tau)=\frac{1}{G^{\prime}(R(t))} \int_{0}^{1} G^{\prime}\left(s\left(w_{\theta \theta}+w\right)(\theta, t)+R(t)\right) d s
\end{array}\right.
$$

By assumption $(* 2)$, we have $0<\frac{1}{\lambda} \leq A(\theta, \tau) \leq \lambda$ for all $(\theta, \tau) \in S^{1} \times[0, \infty)$ for some constant $\lambda$ independent of $(\theta, \tau)$. Thus equation (9) is now uniformly parabolic 
with the estimate

$$
|w(\theta, \tau)|, \quad\left|w_{\theta}(\theta, \tau)\right|, \quad\left|w_{\theta \theta}(\theta, \tau)\right|, \quad\left|\frac{\partial w}{\partial \tau}(\theta, \tau)\right| \leq C,
$$

where $C$ is a positive constant independent of $(\theta, \tau)$. However, since $A(\theta, t)$ depends on $w_{\theta \theta}$, equation (9) is still nonlinear. Note in particular that the derivatives (space or time) of $A(\theta, \tau)$ may not be uniformly bounded on $S^{1} \times[0, \infty)$.

Next, let $Y$ be the function space $\left\{c_{1} \cos \theta+c_{2} \sin \theta: c_{1}, c_{2} \in \mathbb{R}, \theta \in S^{1}\right\}$ and set

$$
\rho(\tau)=\inf _{v \in Y}\|w(\cdot, \tau)-v\|_{L^{2}\left(S^{1}\right)}, \quad E(\tau)=\frac{1}{2} \int_{0}^{2 \pi}\left(\left(\frac{\partial w}{\partial \theta}\right)^{2}-w^{2}\right) d \theta, \quad \tau \in[0, \infty) .
$$

One easily sees that $E(\tau)$ is non-increasing in $\tau \in[0, \infty)$ and it will imply $\lim _{\tau \rightarrow \infty} \rho(\tau)=0$. As a result, there are bounded functions $a(\tau), b(\tau)$ such that $\|w(\theta, \tau)-a(\tau) \cos \theta-b(\tau) \sin \theta\|_{L^{2}\left(S^{1}\right)} \rightarrow 0$ as $\tau \rightarrow \infty$. Moreover, since $w_{\theta \theta}(\theta, \tau)$ is uniformly bounded, this convergence is actually valid in the space $C^{1}\left(S^{1}\right)$.

Finally denote by $\mathcal{Z}[u]$ the number of sign changes of a function $u(\theta)$ on $S^{1}$. For any $v \in Y$, the number $\mathcal{Z}[w(\cdot, \tau)-v]$ is non-increasing in $\tau \in(0, \infty)$ since $w(\theta, \tau)-$ $v(\theta)$ is also a solution to equation (9). This property is valid only for 1-dimensional parabolic equations (see Angenent $[\mathrm{ANG}$ ] or Matano $[\mathrm{M}]$ ). As a result of it, we can infer the convergence of $a(\tau)$ and $b(\tau)$ to some constants $a$ and $b$ respectively as $\tau \rightarrow \infty$. The proof is done.

By (7) one can express the center as

$$
(a, b)=\lim _{t \rightarrow T_{\max }} \frac{1}{\pi} \int_{0}^{2 \pi} u(\theta, t)(\cos \theta, \sin \theta) d \theta .
$$

Recall the relation between the support function $U(\theta): S^{1} \rightarrow \mathbb{R}$ and the position vector $X(\theta): S^{1} \rightarrow \mathbb{R}^{2}$ of a convex closed curve $\gamma$, which is

$$
X(\theta)=U(\theta)(\cos \theta, \sin \theta)+U_{\theta}(\theta)(-\sin \theta, \cos \theta), \quad \theta \in S^{1} .
$$

Hence we have (the tangential part and the normal part have the same integral over $S^{1}$ )

$$
\frac{1}{2 \pi} \int_{0}^{2 \pi} X(\theta) d \theta=\frac{1}{\pi} \int_{0}^{2 \pi} U(\theta)(\cos \theta, \sin \theta) d \theta
$$

and we can rephrase (12) as

$$
(a, b)=\lim _{t \rightarrow T_{\max }} \frac{1}{2 \pi} \int_{0}^{2 \pi} X(\theta, t) d \theta .
$$

Thus if we have the existence of the center of expansion, then it is given by the asymptotic average of position vectors of the evolving curves. Intuitively, this is geometrically reasonable.

Remark 7. Note that the existence of the limit

$$
\lim _{t \rightarrow T_{\max }} \frac{1}{\pi} \int_{0}^{2 \pi} u(\theta, t)(\cos \theta, \sin \theta) d \theta=(a, b) \in \mathbb{R}^{2}
$$

does not necessarily imply that $(a, b) \in \mathbb{R}^{2}$ is the center of expansion since it may not imply that $u(\theta, t)$ is asymptotically given by $R(t)+a \cos \theta+b \sin \theta$. From 
the viewpoint of the Fourier series expansion, it could contain terms of the form $a_{n} \cos n \theta+b_{n} \sin n \theta, n \geq 2, n \in \mathbb{N}$. See an example in the next section.

Remark 8. In the interesting special case when the speed function in flow (1) is given by $G(1 / k)=1 / k$, the integral on the left hand side of (16) is independent of time. Hence the average of position vectors of $\gamma(\cdot, t)$ is time-independent and the center of expansion is determined initially, given by

$$
(a, b)=\frac{1}{\pi} \int_{0}^{2 \pi} u_{0}(\theta)(\cos \theta, \sin \theta) d \theta
$$

Clearly the center is interior to $\gamma_{0}$. We shall see below (see Theorem 13) that this holds for general speed $G$ satisfying $(* 1)$ and $(* 2)$.

Now we are ready to use Chow-Gulliver's Aleksandrov reflection result CG] (for a more geometrical statement, see Theorem 2.2 in Chow C]) to estimate the location of the center of expansion $(a, b) \in \mathbb{R}^{2}$.

For the reader's convenience, we review some definitions of terminology (see $[$ ], p. 390) for the plane case. Let $L$ be a line perpendicular to a unit vector $V \in \mathbb{R}^{2}$. There is a constant $C$ such that $\langle L, V\rangle=\{C\}$, where $\langle$,$\rangle is the inner product of$ $\mathbb{R}^{2}$. Denote by

$$
H_{+}(L)=\left\{p \in \mathbb{R}^{2}:\langle p, V\rangle>C\right\} \quad \text { and } \quad H_{-}(L)=\left\{p \in \mathbb{R}^{2}:\langle p, V\rangle<C\right\} .
$$

They are half-planes to both sides of $L$. Let $\gamma \subset \mathbb{R}^{2}$ be an embedded smooth closed curve and let $\gamma^{L}$ be the reflection of $\gamma$ about the line $L$, i.e.,

$$
\gamma^{L}=\{p-2(\langle p, V\rangle-C) V: p \in \gamma\} .
$$

Definition 9. We say that we can reflect $\gamma$ strictly at $(L, V)$ if

$$
\gamma^{L} \cap H_{-}(L) \subset \operatorname{int}(\gamma) \cap H_{-}(L)
$$

and $V \notin T \gamma_{p}$ (the tangent space to $\gamma$ at $p$ ) for any $p \in \gamma \cap L$. Here int $(\gamma)$ denotes the plane region interior to $\gamma$.

Definition 10. We say that we can reflect $\gamma$ strictly up to $(L, V)$ if we can reflect $\gamma$ strictly at $\left(L^{\prime}, V\right)$, where $L^{\prime}$ is any line parallel to $L$ such that $\left\langle L^{\prime}, V\right\rangle \geq C$. In particular, this implies $V \notin T \gamma_{p}$ for any $p \in \gamma \cap \overline{H_{+}(L)}$, where $\overline{H_{+}(L)}$ denotes the closure of the region $H_{+}(L)$.

The theorem by Chow-Gulliver can be applied to a rather general setting. However, in the setting of the flow (1), it says the following:

Theorem 11 (Chow-Gulliver). Let $G:(0, \infty) \rightarrow(0, \infty), G^{\prime}>0$, be an arbitrary smooth function (here it may not satisfy either $(* 1)$ or $(* 2)$, or both). For the flow (11), if we can reflect the convex $\gamma_{0}$ strictly at (respectively, up to) $(L, V)$, then we can reflect $\gamma(\cdot, t)$ strictly at (respectively, up to) $(L, V)$ for all time $t \in\left[0, T_{\max }\right)$.

Since $\gamma_{0}$ is strictly convex and smooth, along each normal direction $\mathbf{N}(\theta)=$ $(\cos \theta, \sin \theta), \theta \in S^{1}$, there exists a unique line $L(\theta)$ perpendicular to $\mathbf{N}(\theta)$, with $L(\theta) \cap \operatorname{int}\left(\gamma_{0}\right) \neq \varnothing$, such that we can reflect $\gamma_{0}$ strictly up to $(\tilde{L}(\theta), \mathbf{N}(\theta))$ for any line parallel to $L(\theta)$ satisfying $\langle\tilde{L}(\theta), \mathbf{N}(\theta)\rangle>\langle L(\theta), \mathbf{N}(\theta)\rangle$, but not beyond $L(\theta)$. Using these lines $L(\theta)$ one can determine a region $\Omega$ strictly interior to $\gamma_{0}$, given by

$$
\Omega=\left\{p \in \mathbb{R}^{2}:\langle p, \mathbf{N}(\theta)\rangle \leq\langle L(\theta), \mathbf{N}(\theta)\rangle \text { for all } \theta \in S^{1}\right\}
$$


Clearly $\Omega$ is a closed set in $\mathbb{R}^{2}$. Moreover it is convex since for $p, q \in \Omega, 0 \leq s \leq$ 1 , we have

$$
\langle s p+(1-s) q, \mathbf{N}(\theta)\rangle=s\langle p, \mathbf{N}(\theta)\rangle+(1-s)\langle q, \mathbf{N}(\theta)\rangle \leq\langle L(\theta), \mathbf{N}(\theta)\rangle
$$

for all $\theta \in S^{1}$. Hence $s p+(1-s) q \in \Omega$ also. By Theorem 13 , we also see that $\Omega$ is not empty as it contains at least the center of expansion.

Remark 12. Analytically, one can describe the boundary of the region $\Omega$ as the envelope $E$ of the family of lines $L(\theta)$, under the assumption that its distance $d(\theta)$ to the origin is a differentiable function. The family of lines $L(\theta)$ is now given by

$$
F(x, y, \theta):=x \cos \theta+y \sin \theta-d(\theta)=0, \quad(x, y) \in \mathbb{R}^{2},
$$

where $\theta \in S^{1}$ is viewed as a parameter. It is known that the envelope $E$ is the collection of all points $(x, y) \in \mathbb{R}^{2}$ satisfying the following system of equations (see the book by Courant and John [CJ], p. 293):

$$
\left\{\begin{array}{l}
F(x, y, \theta)=x \cos \theta+y \sin \theta-d(\theta)=0, \\
F_{\theta}(x, y, \theta)=-x \sin \theta+y \cos \theta-d_{\theta}(\theta)=0 .
\end{array}\right.
$$

Hence we obtain $E=\left\{(x(\theta), y(\theta)): \theta \in S^{1}\right\}$, where

$$
(x(\theta), y(\theta))=d(\theta)(\cos \theta, \sin \theta)+d_{\theta}(\theta)(-\sin \theta, \cos \theta), \quad \theta \in S^{1} .
$$

Note that (22) coincides with (13).

We can now prove the following:

Theorem 13. Let $G:(0, \infty) \rightarrow(0, \infty), G^{\prime}>0$, be an arbitrary smooth function satisfying $(* 1)$ and $(* 2)$ (so that the center exists). Then under the flow (10), the center of expansion lies on $\Omega$.

Proof. For a fixed $\theta_{0} \in S^{1}$, we may assume $\mathbf{N}\left(\theta_{0}\right)=(0,1)$ and the unique line $L\left(\theta_{0}\right)$ is the line $y=0$ (i.e., $x$-axis). Also choose the origin of $\mathbb{R}^{2}$ at some point $O \in L\left(\theta_{0}\right) \cap \operatorname{int}\left(\gamma_{0}\right)$. By Theorem [11, for any $\varepsilon>0$ we have

$$
\gamma^{\tilde{L}}(\cdot, t) \cap H_{-}(\tilde{L}) \subset \operatorname{int}(\gamma(\cdot, t)) \cap H_{-}(\tilde{L}),
$$

where $\tilde{L}$ is the line $\tilde{L}=\{y=\varepsilon>0\}$ and moreover $(0,1) \notin T \gamma(\cdot, t)_{p}$ for all $p \in$ $\gamma(\cdot, t) \cap \overline{H_{+}(\tilde{L})}$ for all $t \in\left[0, T_{\max }\right)$. By the inclusion relation (23) we have

$$
\left\langle\frac{1}{2 \pi} \int_{0}^{2 \pi}[X(\theta, t)-(0, \varepsilon)] d \theta,(0,1)\right\rangle=\frac{1}{2 \pi} \int_{0}^{2 \pi}\langle X(\theta, t)-(0, \varepsilon),(0,1)\rangle d \theta \leq 0
$$

for all $t \in\left[0, T_{\max }\right)$, where the position vector $X(\theta, t)$ is with respect to the origin $O$. Letting $t \rightarrow T_{\max }$ and $\varepsilon \rightarrow 0$ in (24), by (15)) we obtain $\langle(a, b),(0,1)\rangle \leq 0$, which means that the center $(a, b)$ is on or below the $x$-axis. As this property is valid for any direction $\theta_{0} \in S^{1}$, we must have $(a, b) \in \Omega$.

Remark 14. Intuitively, one can also argue as follows. Suppose the center $(a, b)$ is above the $x$-axis with $b=\delta>0$. In light of the $C^{1}$ convergence (7), $\gamma(\cdot, t)$ is asymptotically a circle as $t \rightarrow T_{\max }$. Hence, as $t$ is close to $T_{\max }, \gamma^{\tilde{L}}(\cdot, t) \cap H_{-}(\tilde{L})$ will not be contained in $\operatorname{int}(\gamma(\cdot, t)) \cap H_{-}(\tilde{L})$, where $\tilde{L}$ is the line $\{y=\varepsilon>0\}$ with $\varepsilon<\delta$. This is in contradiction to (23). Hence we must have $b \leq 0$. 


\section{NONEXistence OF CENTER OF EXPANSION}

We first recall some relations between the support function and the Hausdorff distance. Let $K$ and $L$ be two compact subsets in $\mathbb{R}^{n}$. Their Hausdorff distance is defined as

$$
\delta(K, L)=\max \left\{\sup _{\theta \in K} \inf _{y \in L}|\theta-y|, \quad \sup _{\theta \in L} \inf _{y \in K}|\theta-y|\right\} .
$$

We have the following result (see Schneider $[\underline{\mathrm{S}}$, p. 53):

Lemma 15. Assume $K$ and $L$ are two convex bodies in $\mathbb{R}^{n}$. Then

$$
\delta(K, L)=\sup _{\theta \in S^{n-1}}\left|U_{K}(\theta)-U_{L}(\theta)\right|,
$$

where $U_{K}(\theta)$ and $U_{L}(\theta)$ are the support functions of $K$ and $L$ respectively.

Thus if two families of expanding convex closed curves have their support functions stay close to each other, their Hausdorff distance is small. This also implies that their length and enclosed area are close to each other.

In this section, we shall use a specific example to show that the expanding curve $\gamma(\cdot, t)$ does not have a center of expansion if $G(z)$ does not satisfy both assumptions $(* 1)$ and $(* 2)$. Moreover, we can make the asymptotic average of position vectors of $\gamma(\cdot, t)$ as close to the origin $(0,0)$ as we want. But no matter how close it is, it is still far away from the existence of a center of expansion. See Theorem 2.7 of [T2] and Yagisita [Y] also.

Take $G(z)=1-e^{-z}, z \in(0, \infty), G^{\prime}>0$ everywhere. For such a $G$ the assumption $(* 1)$ is not satisfied (but $(* 2)$ is still satisfied). Under the flow (1), the equation for the support function $u(\theta, t)$ is given by

$$
\frac{\partial u}{\partial t}(\theta, t)=1-e^{-\left(u_{\theta \theta}+u\right)} .
$$

Let $r(t)$ be the solution to the ODE

$$
\frac{d r}{d t}(t)=1-e^{-r(t)}, \quad r(0)=1 .
$$

Such an $r(t)$ is clearly defined on $t \in[0, \infty)$ with $r(\infty)=\infty$. Set the new time $\tau$ as

$$
\tau=\log \left(\frac{1-e^{-r(t)}}{1-e^{-r(0)}}\right), \quad t \in[0, \infty) .
$$

We have $\tau(0)=0$ and $\lambda:=\tau(\infty)>0$ is a finite positive number, $\lambda=\log [e /(e-1)]$.

If we let $w(\theta, t)=u(\theta, t)-r(t)$, then in terms of the new time $\tau$ it satisfies the equation

$$
\frac{\partial w}{\partial \tau}(\theta, \tau)=\frac{\left[1-e^{-\left(w_{\theta \theta}(\theta, \tau)+w(\theta, \tau)+r(t)\right)}\right]-\left[1-e^{-r(t)}\right]}{e^{-r(t)}}=1-e^{-\left[w_{\theta \theta}(\theta, \tau)+w(\theta, \tau)\right]},
$$

which happens to be the same as the original equation (27).

For a given sufficiently small $\varepsilon>0$, choose $T>0$ such that $\tau(T)=\lambda-\varepsilon / 50$ and let

$w^{*}(\theta, \tau)=\cos 2 \theta+100\left[\tau-\left(\lambda-\frac{\varepsilon}{50}\right)\right], \quad w_{*}(\theta, \tau)=\cos 2 \theta-100\left[\tau-\left(\lambda-\frac{\varepsilon}{50}\right)\right]$, 
where $(\theta, \tau) \in S^{1} \times[\lambda-\varepsilon / 50, \lambda):=I$. We have

$\left|1-e^{-\left[w_{\theta \theta}^{*}(\theta, \tau)+w^{*}(\theta, \tau)\right]}\right| \leq 100$ and $\quad\left|1-e^{-\left[w_{* \theta \theta}(\theta, \tau)+w_{*}(\theta, \tau)\right]}\right| \leq 100, \quad(\theta, \tau) \in I$.

Hence $w^{*}$ and $w_{*}$ are both super-solution and sub-solution to the equation (29) on $(\theta, \tau) \in I$.

Let $u(\theta, t)$ be the solution to equation (27) on $S^{1} \times[T, \infty)$ with the initial condition at time $T$ given by $u(\theta, T)=r(T)+\cos 2 \theta$. Then the function $w(\theta, t)=$ $u(\theta, t)-r(t), t \in[T, \infty)$, in terms of the variable $(\theta, \tau)$, is a solution to (29) on $(\theta, \tau) \in I$ with $w(\theta, \lambda-\varepsilon / 50)=\cos 2 \theta$ and by the maximum principle we obtain

$$
\cos 2 \theta-2 \varepsilon \leq w_{*}(\theta, \tau) \leq w(\theta, \tau) \leq w^{*}(\theta, \tau) \leq \cos 2 \theta+2 \varepsilon
$$

on $I$, which implies that

$$
(r(t)+\cos 2 \theta)-2 \varepsilon \leq u(\theta, t) \leq(r(t)+\cos 2 \theta)+2 \varepsilon
$$

on $(\theta, t) \in S^{1} \times[T, \infty)$.

Remark 16. Note the difference: $u(\theta, t)$ is an unbounded solution of equation (27) on the infinite time interval $t \in[T, \infty)$, whereas $w(\theta, \tau)$ is a bounded solution of the same equation (29) on the finite time interval $\tau \in[\lambda-\varepsilon / 50, \lambda)$.

The geometric meaning of the above is that at time $T$ one can use the support function $u(\theta, T)=r(T)+\cos 2 \theta$ to construct a smooth convex closed curve $\gamma(\cdot, T)$ with curvature

$$
k(\theta, T)=\frac{1}{u_{\theta \theta}(\theta, T)+u(\theta, T)}=\frac{1}{r(T)-3 \cos 2 \theta}>0, \quad \theta \in S^{1}
$$

and evolve it under the expanding flow. Then the support function $u(\theta, t)$ of $\gamma(\cdot, t)$ will satisfy the estimate (30). By Lemma 15, for sufficiently small $\varepsilon>0$, the isoperimetric difference $L^{2}-4 \pi A$ of $\gamma(\cdot, t)$ will be close to the isoperimetric difference of the convex curve with support function $r(t)+\cos 2 \theta$ for $t \in[T, \infty)$. However, the isoperimetric difference of the latter is a fixed positive constant independent of time, given by

$$
\begin{aligned}
& L^{2}(t)-4 \pi A(t) \\
& =\left[\int_{0}^{2 \pi}(r(t)+\cos 2 \theta) d \theta\right]^{2}-2 \pi \int_{0}^{2 \pi}\left[(r(t)+\cos 2 \theta)^{2}-(r(t)+\cos 2 \theta)_{\theta}^{2}\right] d \theta \\
& =6 \pi^{2} \text { for all } t \in[T, \infty) .
\end{aligned}
$$

Hence the isoperimetric difference of the former decreases to a positive constant (close to $6 \pi^{2}$ ) as $t \rightarrow \infty$ and there is no center of expansion for $\gamma(\cdot, t)$ (see Remark (6). This also says that if $u(\theta, t)$ satisfies (30) on $S^{1} \times[T, \infty)$, then it is impossible for it to satisfy an estimate of the form (7).

If we choose $\varepsilon$ to be even smaller, then $T$ will become larger and $u(\theta, t)$ will be even closer to $r(t)+\cos 2 \theta$ on $S^{1} \times[T, \infty)$, with the isoperimetric difference of $\gamma(\cdot, t)$ even closer to the constant $6 \pi^{2}$. On the other hand, by (30) we can infer that

$$
\frac{1}{\pi} \int_{0}^{2 \pi} u(\theta, t)(\cos \theta, \sin \theta) d \theta \in\left[-\frac{8 \varepsilon}{\pi}, \frac{8 \varepsilon}{\pi}\right] \times\left[-\frac{8 \varepsilon}{\pi}, \frac{8 \varepsilon}{\pi}\right], \quad t \in[T, \infty),
$$

which means that the average of position vectors of $\gamma(\cdot, t)$ is even closer to the origin $(0,0)$ for $t \in[T, \infty)$. Thus from this example we may say that, in general, the 
concept of the center of expansion is not the same as that of the asymptotic average of the position vector. See Remark 7 also.

\section{ACKNOWLEDGMENTS}

The second author would like to express his gratitude to Professor Bennett Chow of the University of California, San Diego, for being a long-time friend and mentor. We are also grateful to the referee for pointing out some errors and for some valuable comments. In particular, one of them leads to Remark 2 ,

\section{REFERENCES}

[A] B. Andrews, Evolving convex curves, Cal. of Var. \& PDEs, 7 (1998), no. 4, 315-371. MR.1660843(99k:58038)

[ANG] S. Angenent, The zero set of a solution of a parabolic equation, J. für die reine and angewandte Mathematik, 390 (1988), 79-96. MR 953678 (89j:35015)

[C] B. Chow, Geometric aspects of Aleksandrov reflection and gradient estimates for parabolic equations, Comm. Anal. \& Geom., 5 (1997), no. 2, 389-409. MR1483984 (98k:53045)

[CG] B. Chow; R. Gulliver, Aleksandrov reflection and nonlinear evolution equations, I: The n-sphere and n-ball, Cal. of Var. \& PDEs, 4 (1994), 249-264. MR1386736 (97f:53064)

[CJ] R. Courant; F. John, Introduction to Calculus and Analysis, Vol. II, John Wiley \& Sons, 1974; reprint of the 1974 edition, Springer-Verlag, 1989. MR.1016380 (90j:00002b)

[CLT] B. Chow; L.-P. Liou; D.-H. Tsai, Expansion of embedded curves with turning angle greater than $-\pi$, Invent. Math., 123 (1996), 415-429. MR1383955 (97c:58025)

[CT] B. Chow; D.-H. Tsai, Geometric expansion of convex plane curves, J. of Diff. Geom., 44 (1996), 312-330. MR.1425578 (97m:58041)

[GH] M. Gage; R. Hamilton, The heat equation shrinking convex plane curves, J. of Diff. Geom., 23 (1986), 69-96. MR840401 (87m:53003)

[M] H. Matano, Nonincrease of the lap-number of a solution for a one-dimensional semilinear parabolic equation, J. Fac. Sci. Univ. Tokyo Sec. IA Math., 29 (1982), 401-441. MR672070 (84m:35060)

[S] R. Schneider, Convex Bodies: The Brunn-Minkowski Theory, Cambridge University Press, 1993. MR 1216521 (94d:52007)

[T1] D.-H. Tsai, Geometric expansion of starshaped plane curves, Comm. Anal. \& Geom., 4 (1996), no. 3, 459-480. MR.1415752 (97k:58042)

[T2] D.-H. Tsai, Asymptotic closeness to limiting shapes for expanding embedded plane curves, Invent. Math., 162 (2005), 473-492. MR2198219 (2006j:53099)

[T3] D.-H. Tsai, Behavior of the gradient for solutions of parabolic equations on the circle, Cal. of Var. \& PDEs, 23 (2005), 251-270. MR.2142063(2006d:35116)

[U] J. Urbas, An expansion of convex hypersurfaces, J. of Diff. Geom., 33 (1991), 91-125. Correction, ibid., 35 (1992), 763-765. MR1085136 (91j:58155) MR1163459 (93b:58142)

[Y] H. Yagisita, Asymptotic behaviors of star-shaped curves expanding by $V=1-K$, Diff. \& Integ. Eqs., 18 (2005), no. 2, 225-232. MR2106103 (2005m:53128)

Department of Mathematics, National Tsing Hua University, Hsinchu 30013, Taiwan E-mail address: yclin@math.nthu.edu.tw

Department of Mathematics, National Tsing Hua University, Hsinchu 30013, Taiwan

E-mail address: dhtsai@math.nthu.edu.tw 